\title{
Screening effect on electron capture in presupernova stars
}

\author{
M. Q. Liu, J. Zhang, and Z. Q. Luo
}

\author{
Institute of Theoretical Physics, China West Normal University, Nanchong 637002, PR China \\ e-mail: Liumq@cwnu.edu.cn; menquanliu@126.com
}

Received 25 May 2006 / Accepted 12 October 2006

ABSTRACT

\begin{abstract}
Aims. With the new electron capture results from the improved GT resonance distribution, we analyzed the electron screening effect on electron capture at the presupernova stage.

Methods. The relativistically degenerate electron liquid screening potential derived from the linear response theory are adopted. The calculation of the electron capture rates is based on the shell-model. Both the change of electron energy and the shift in the threshold for electron capture in screening are taken into account.

Results. The results indicate that the influence of electron screening on the electron capture is prominent in high-density matter. As the improved electron capture rate is considered, screening effect on the electron capture rate increases by about 1 percent at $\rho Y_{\mathrm{e}}>$ $10^{9} \mathrm{~g} / \mathrm{cm}^{3}$.

Conclusions. The influence of screening on the electron capture reaction is about 10 percent for ${ }^{56} \mathrm{Co}$ at $\rho Y_{\mathrm{e}} \approx 10^{9} \mathrm{~g} / \mathrm{cm}^{3}$. Screening may affect the structure and evolution of presupernova and should be included in any precise simulation.
\end{abstract}

Key words. supernovae: general - nuclear reactions, nucleosynthesis, abundances - methods: numerical

\section{Introduction}

The electron capture process plays a large role in astrophysics because it is a key process in the evolution of presupernova star and the explosion of supernova (SN, Woosley et al. 2002). In this process, the electron number density decreases, and the electron degenerate pressure decreases accordingly. One of the products in the capture process, the neutrinos, escape and take plenty of energy away from the star when the density is below the trapped density of the neutrino. Both the decrease in degenerate pressure and the loss of energy accelerate the collapse (Bethe et al. 1979, 1990) of the star.

The weak interaction processes, including the electron capture and $\beta$ decay, in dense matter have been investigated extensively in last decades. Based on the simple shell model, Fuller et al. (1982, hereafter FFN) accomplished pioneering work, in which they discussed the location and the strength of the Gamow-Teller (GT) resonance transition and made detailed calculations of 226 nuclei with mass number A ranging from 21 to 60. Auferheide et al. (1994) pointe out that the nuclei with $A>60$ should also be taken into account in the theoretical analysis because the core region of presupernova is mainly composed of neutron-rich nuclei. They extended FFN's work to some nuclei with $A>60$ and provided the weak interaction rates of the 150 most abundant isotopes with some key temperatures and densities at the presupernova stage. In recent years, the largescale shell-model calculations and the shell-model Mont Carlo calculations (SMMC) of the GT strength distribution were done by Langanke \& Martinez-Pinedo (1997, 2000, hereafter LMP). Their results indicate that the improved electron capture rates are significantly lower than usually adopted in core collapse calculation.

People have also addressed the effect of the electron screening in a weak interaction. In the 1950s, Reitz (1950) analyzed the effect of screening on $\beta$ decay by solving the Dirac equation and concluded that the screening slightly decreases the $\beta$ decay rate. After that, the screening effect on Fermi function and Coulomb correction in $\beta$ decay were considered (Chen et al. 1966; Matese et al. 1966). Gutierrez (1996) suggested that the decrease in electron Fermi energy is equal to the shift in the chemical potential of nuclei before and after the capture reaction. Luo et al. (1996, 2001) argue that the screening also affects the phase space distribution of the electron besides the Fermi energy. The calculation showed that the screening can reduce the rate of change of electron fraction by $10-20 \%$. With the same method as Gutierrez, Bravo (1999) solved the nuclear statistical equation and found that the neutronization rate is higher than $28 \%$ at some temperatures and densities.

Numerical simulations show that the $\mathrm{SN}$ explosion depends on the persupernova structure and the evolution mode, which is especially sensitive to the physical parameters' input. The parameters are closely related to the electron fraction and the weak interaction rates at the presupernova stage (Heger et al. 2001). Therefore, it is imperative to calculate the electron capture rates with high precision. In this paper, the screening effects on the electron capture rates for the improved results from Langanke et al. (1999) are evaluated.

\section{Electron capture rate in screening}

For the convenience of explaining the results, it is necessary to briefly introduce the formulae of our calculations. The capture rate for the $k$ th nucleus $(Z, A)$ in thermal equilibrium at temperature $T$ is given by a sum over the initial parent states $i$ and the final daughter states $f$ (Pruet et al. 2003),

$\lambda_{k}=\sum_{i} \frac{\left(2 J_{i}+1\right) \mathrm{e}^{-E_{i} / k_{\mathrm{B}} T}}{G(Z, A, T)} \sum_{f} \lambda_{i f}$, 
where $J_{i}$ and $E_{i}$ are the spin and excitation energy of the parent states, $k_{\mathrm{B}}$ the Boltzmann factor, $G(Z, A, T)$ the nuclear partition function, $\lambda_{i f}$ the electron capture rate from one of the initial states to all possible final states, $\lambda_{i f}=\frac{\ln 2}{(f t)_{i f}} f_{i f}$ with the relation $\frac{1}{(f t)_{i f}}=\frac{1}{(f t)_{i f}^{F}}+\frac{1}{(f t)_{i f}^{G T}}$. The $f t$-values and the corresponding GT or Fermi transition matrix elements are related to the following equations,

$$
\frac{1}{(f t)_{i f}^{\mathrm{GT}}} \approx \frac{10^{3.59}}{\left|M_{\mathrm{GT}}\right|_{i f}^{2}}, \frac{1}{(f t)_{i f}^{\mathrm{F}}} \approx \frac{10^{3.79}}{\left|M_{\mathrm{F}}\right|_{i f}^{2}} .
$$

The phase space integral $f_{i f}$ is defined as

$$
f_{i f}=\int_{w}^{\infty} \varepsilon^{2}\left(Q_{i f}+\varepsilon\right)^{2} G(\mathrm{Z}, \varepsilon) f_{\mathrm{e}} \mathrm{d} \varepsilon
$$

where $\varepsilon$ is the electron energy (the energy is in unit of $m_{\mathrm{e}} c^{2}$ in this paper except when explicitly written with the unit $\mathrm{MeV}$ ), $f_{\mathrm{e}}$ is the Fermi-Dirac distribution function of the electrons, and $G(Z, \varepsilon)$ is the Coulomb correction factor. The nuclear energy difference between the parent and daughter nuclei $Q_{i f}=$ $\left(M_{\mathrm{p}}-M_{\mathrm{d}}\right) c^{2}+E_{i}-E_{f}$, where $M_{\mathrm{p}}$ and $M_{\mathrm{d}}$ are the mass of the parent and daughter nucleus, respectively, and $E_{f}$ is the excitation energy of daughter state. Without considering the screening, the threshold energy $w$ equals the static energy of an electron if the energy difference $Q_{i f}$ is less than the static energy of an electron; otherwise it is equal to $\left|Q_{i f}\right|$.

The screening mainly affects the electron capture in three facets. (1) The screening changes the Coulomb in-wave function of the electron, which, however, can be neglected because the screening potential $D$ is much less than the average energy of the electrons. (2) The screening decreases the electron energy $\varepsilon$ in the capture reaction to $\varepsilon^{\prime}=\varepsilon-D$. (3) The screening relatively decreases the number of the high energy electrons whose energy is more than the threshold energy of electron capture. Thus, the threshold energy will increase from $w$ to $w_{S}=w+\Delta Q$, where $\Delta Q$ represents the change in binding energy of the final and initial nuclei due to the presence of the electron gas. In the plasma, a nucleus has its binding energy increased due to interactions with the dense electron gas. In the Wigner-Seitz approximation, the extra binding of an atom can be written as $1.764 \times 10^{-5} Z^{5 / 3}\left(\rho Y_{\mathrm{e}}\right)^{1 / 3} \mathrm{MeV}$ (Salpeter et al. 1969). Because of the charge dependence of this binding, the effective nuclear $Q$-value changes at high density. In general, the average $Q$-value for each electron will increase by (FFN 1982)

$\Delta Q \approx 2.94 \times 10^{-5} Z^{2 / 3}\left(\rho Y_{\mathrm{e}}\right)^{1 / 3} \mathrm{MeV}$,

where $Z$ is the charge of nucleus, $\rho$ the mass density and $Y_{\mathrm{e}}$ the electron fraction. Thus the phase space integral with screening is written as

$f_{i f}^{S}=\int_{w_{S}}^{\infty} \varepsilon^{\prime 2}\left(Q_{i f}+\varepsilon^{\prime}\right)^{2} G\left(Z, \varepsilon^{\prime}\right) f_{\mathrm{e}} \mathrm{d} \varepsilon^{\prime}$.

Then, the key point in the calculation that includes the screening is to calculate the screening potential $D$. Recently, Itoh et al. (2002) used the linear response theory to calculate the screening potential caused by relativistically degenerate electron. This theory is widely used in the field of solid-state physics and liquid physics. Their results provide a more precise screening potential,

$D=7.525 \times 10^{-3} Z\left(\frac{Z}{A} \rho_{6}\right)^{1 / 3} J\left(r_{\mathrm{s}}, R\right) \mathrm{MeV}$,

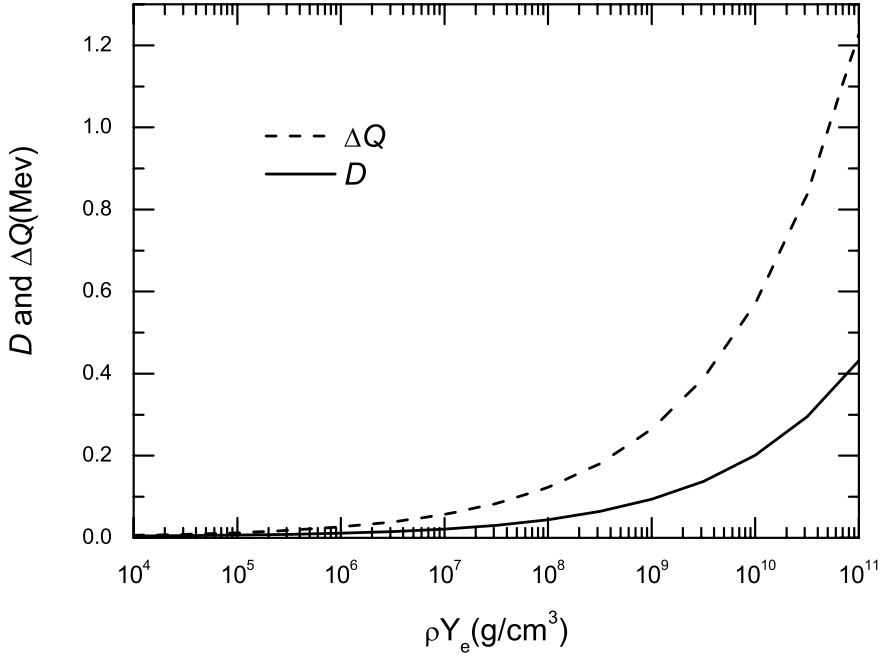

Fig. 1. $D$ and $\Delta Q$ as a function of density $\left(Y_{\mathrm{e}}=0.45\right)$.

Table 1. The effect of electron screening on ${ }^{56} \mathrm{Co}\left(\mathrm{e}^{-}, v_{\mathrm{e}}\right){ }^{56} \mathrm{Fe} .(T=$ $\left.5 \times 10^{9} \mathrm{~K}, Y_{\mathrm{e}}=0.45\right), C_{1}$ denotes the screening factor in the fiducial GT resonance distribution, $C_{2}$ denotes the screening factor with the improved GT resonance distribution.

\begin{tabular}{lllrr}
\hline \hline $\log _{10}\left(\rho Y_{\mathrm{e}}\right)$ & $\lambda_{1}\left(\mathrm{~s}^{-1}\right)$ & $\lambda_{2}\left(\mathrm{~s}^{-1}\right)$ & $C_{1}$ & $C_{2}$ \\
\hline 7.00 & $2.69 \mathrm{E}-2$ & $1.11 \mathrm{E}-2$ & 0.9516 & 0.9521 \\
7.50 & $1.06 \mathrm{E}-1$ & $3.69 \mathrm{E}-2$ & 0.9374 & 0.9453 \\
8.00 & $5.49 \mathrm{E}-1$ & $1.31 \mathrm{E}-1$ & 0.9214 & 0.9396 \\
8.50 & $3.64 \mathrm{E}+0$ & $5.28 \mathrm{E}-1$ & 0.9102 & 0.9303 \\
9.00 & $2.74 \mathrm{E}+1$ & $3.28 \mathrm{E}+0$ & 0.9062 & 0.8965 \\
9.50 & $2.09 \mathrm{E}+2$ & $4.11 \mathrm{E}+1$ & 0.9061 & 0.8812 \\
10.00 & $1.55 \mathrm{E}+3$ & $4.91 \mathrm{E}+2$ & 0.9072 & 0.8905 \\
10.50 & $1.11 \mathrm{E}+4$ & $4.71 \mathrm{E}+3$ & 0.9083 & 0.8981 \\
11.00 & $7.77 \mathrm{E}+4$ & $3.94 \mathrm{E}+4$ & 0.9095 & 0.9032 \\
\hline
\end{tabular}

where $\rho_{6}$ is the density in unit of $10^{6} \mathrm{~g} / \mathrm{cm}^{3}, J\left(r_{s}, R\right)=$ $\sum_{i, j=0}^{10} a_{i j} s^{i} u^{j}, s=\frac{1}{2}\left(\log _{10} r_{s}+3\right), u=\frac{1}{25}(R-25), R=2 k_{\mathrm{F}} r_{\mathrm{nuc}}=$ $6.3 \times 10^{-3} Z^{1 / 3} \rho_{6}{ }^{1 / 3}$, and $r_{s}=1.388 \times 10^{-2}\left(\frac{A}{Z \rho_{6}}\right)^{1 / 3}$. One can find the factor $a_{i j}$ in Itoh et al. (2002), and $r_{\text {nuc }}$ is the nuclear radius and $k_{\mathrm{F}}$ the electron Fermi wave number, $k_{\mathrm{F}}=2.613 \times$ $10^{10}\left(\frac{A}{Z \rho_{6}}\right)^{1 / 3}$. Formula (6) is valid for $10^{-5} \leq r_{s} \leq 10^{-1}$ and $0 \leq R \leq 50$, which are usually fulfilled in the presupernova environment.

Figure 1 shows the variation in $D$ and $\Delta Q$ for ${ }^{56} \mathrm{Co}$ with $Y_{\mathrm{e}}=$ 0.45 . From Fig. 1, one can see that both corrections increase with density, and the correction of threshold energy $\Delta Q$ is more obvious than $D$ at high density. Therefore, the electron capture rate with a low threshold energy will be easier to change in the high density environment of presupernova.

Provided the excited state distribution of nucleus is known, the electron capture rates can be obtained with high precision. Unfortunately, the nuclei in the presupernova environment are usually unstable, and the distribution of the high excited states are almost continuous (particularly for the heavier nuclei), so it is difficult to get an explicit distribution for each nucleus. Following the technique of Auderheide et al. (1994), we employed the so-called Brink hypothesis and assumed that the distribution of the parent excited states is similar to that of the ground state and that the location of the GT resonance moves upward in the daughter nucleus by the same amount.

People can split the sum of final states of daughter nuclei into two parts for convenience. One is the contribution $\lambda_{0}$ from 
Table 2. The effect of electron screening on ${ }^{56} \mathrm{Co}\left(\mathrm{e}^{-}, v_{\mathrm{e}}\right)^{56} \mathrm{Fe}$, where the left panel is the results of $\left(\rho=2.33 \times 10^{9} \mathrm{~g} / \mathrm{cm}^{3}, Y_{\mathrm{e}}=0.43, D=0.095 \mathrm{MeV}\right.$, $\Delta Q=0.265 \mathrm{MeV}$ and the right panel is the results of $\left(\rho=2.44 \times 10^{10} \mathrm{~g} / \mathrm{cm}^{3}, Y_{\mathrm{e}}=0.41, D=0.208 \mathrm{MeV}, \Delta Q=0.574 \mathrm{MeV}\right)$. The other notes are the same as that in Table 1.

\begin{tabular}{l|llrr|lrrr}
\hline \hline$T_{9}\left(10^{9} \mathrm{~K}\right)$ & $\lambda_{1}\left(\mathrm{~s}^{-1}\right)$ & $\lambda_{2}\left(\mathrm{~s}^{-1}\right)$ & $C_{1}$ & $C_{2}$ & $\lambda_{1}\left(\mathrm{~s}^{-1}\right)$ & $\lambda_{2}\left(\mathrm{~s}^{-1}\right)$ & $C_{1}$ & $C_{2}$ \\
\hline 1 & $2.26 \mathrm{E}+1$ & $2.30 \mathrm{E}+0$ & 0.8990 & 0.8956 & $1.47 \mathrm{E}+3$ & $4.57 \mathrm{E}+2$ & 0.9053 & 0.8872 \\
2 & $2.31 \mathrm{E}+1$ & $2.39 \mathrm{E}+0$ & 0.9001 & 0.8957 & $1.48 \mathrm{E}+3$ & $4.59 \mathrm{E}+2$ & 0.9055 & 0.8876 \\
3 & $2.38 \mathrm{E}+1$ & $2.57 \mathrm{E}+0$ & 0.9019 & 0.8958 & $1.49 \mathrm{E}+3$ & $4.64 \mathrm{E}+2$ & 0.9058 & 0.8881 \\
4 & $2.49 \mathrm{E}+1$ & $2.81 \mathrm{E}+0$ & 0.9042 & 0.8965 & $1.50 \mathrm{E}+3$ & $4.70 \mathrm{E}+2$ & 0.9062 & 0.8889 \\
5 & $2.64 \mathrm{E}+1$ & $3.15 \mathrm{E}+0$ & 0.9070 & 0.8981 & $1.52 \mathrm{E}+3$ & $4.78 \mathrm{E}+2$ & 0.9068 & 0.8899 \\
6 & $2.83 \mathrm{E}+1$ & $3.60 \mathrm{E}+0$ & 0.9102 & 0.9005 & $1.53 \mathrm{E}+3$ & $4.87 \mathrm{E}+2$ & 0.9074 & 0.8910 \\
7 & $3.06 \mathrm{E}+1$ & $4.18 \mathrm{E}+0$ & 0.9136 & 0.9038 & $1.56 \mathrm{E}+3$ & $4.99 \mathrm{E}+2$ & 0.9082 & 0.8924 \\
8 & $3.33 \mathrm{E}+1$ & $4.91 \mathrm{E}+0$ & 0.9171 & 0.9076 & $1.58 \mathrm{E}+3$ & $5.13 \mathrm{E}+2$ & 0.9091 & 0.8938 \\
9 & $3.66 \mathrm{E}+1$ & $5.82 \mathrm{E}+0$ & 0.9206 & 0.9118 & $1.62 \mathrm{E}+3$ & $5.28 \mathrm{E}+2$ & 0.9100 & 0.8955 \\
10 & $4.05 \mathrm{E}+1$ & $6.95 \mathrm{E}+0$ & 0.9242 & 0.9161 & $1.65 \mathrm{E}+3$ & $5.46 \mathrm{E}+2$ & 0.9111 & 0.8972 \\
\hline
\end{tabular}

the low-energy region near the ground state and the other is the contribution $\lambda_{\mathrm{GT}}$ from the resonance region dominated by the GT resonance transition. The GT strength distribution is similar to a Gaussian distribution (Kar et al. 1994) and the GT resonance energy $E_{\mathrm{GT}}=\Delta E_{s p}+\Delta E_{p h}+\Delta E_{\text {pair }}$, where $\Delta E_{s p}$ is the energy difference between the new occupied neutron orbital and the ground state; $\Delta E_{p h}$ is the repulsion energy that should be supplied to pull the neutron out of the daughter ground state; $\Delta E_{\text {pair }}$ is the cost of breaking a neutron pair in daughter nucleus with an even number of neutrons. The resonance transition matrix element $\left|M_{\mathrm{GT}}\right|^{2}$ can be derived from the model calculation, such as the simple shell-model calculation (FFN 1982). With Auderheide's method, we take $E_{\mathrm{GT}}=5.381 \mathrm{MeV},\left|M_{\mathrm{GT}}\right|^{2}=6$ (quenching factor is 0.5 ) for ${ }^{56} \mathrm{Co}$ in this paper.

LMP adopted the shell-model diagonalization in the $p f$ shell with modified KB3 interaction to calculate the electron capture rates of six important odd-odd nuclei in LMP (1999). They find that the GT centroids $E_{\mathrm{GT}}$ is typically $2 \mathrm{MeV}$ higher and the resonance transition matrix element decreases more than the previous estimation. These changes make the electron capture rates 1 order of magnitude too low compared to the fiducial results (Aufderheide et al. 1994; FFN 1982) except for ${ }^{58} \mathrm{Mn}$. More detailed large-scale shell-model calculations by LMP show that the electron capture rates are significantly lower for nuclei with the mass number ranging from 45 to 65 in the presupernova environment. For example, the electron capture rate of ${ }^{56} \mathrm{Co}$ is $1.7 \times 10^{-3} \mathrm{~s}^{-1}$ by FFN and is $1.2 \times 10^{-4} \mathrm{~s}^{-1}$ by LMP at temperature $T=10^{9} \mathrm{~K}$ and density $\rho Y_{\mathrm{e}}=10^{7} \mathrm{~g} / \mathrm{cm}^{3}$ (FFN 1982b; LMP 2000). However, the effect of screening on the electron capture is ignored in these calculations.

We here calculate the electron capture rates with screening for capture reaction of ${ }^{56} \mathrm{Co}$, which is one of the most important electron capturing nuclei in presupernova. We take $E_{\mathrm{GT}}$ as $8.2 \mathrm{MeV}$ and $\left|M_{\mathrm{GT}}\right|^{2}$ as 7.7 in the improved method derived by LMP (1999). In addition, the quenching factor changes from 0.5 to 0.55 . For a comparison,we define a screening factor, a ratio $C$ between the electron capture rates with $\left(\lambda^{\prime}\right)$ and without $(\lambda)$ screening, $C=\lambda^{\prime} / \lambda$.

We show the influence of electron screening on the reaction ${ }^{56} \mathrm{Co}\left(\mathrm{e}^{-}, v_{\mathrm{e}}\right){ }^{56} \mathrm{Fe}$ at different temperatures, densities, and electron fraction in Tables 1 and 2, where $\lambda_{1}$ is the electron capture rate in the fiducial GT resonance distribution and $\lambda_{2}$ the electron capture rate with the improved GT resonance distribution. Although the modified rates obviously decrease, they are not as low as LMP's results. The reason is that LMP handle the ground state $(J=4)$ and the first excited states $(J=$ 3,5 and 1) of parent nucleus, respectively. Their calculation adopts 33 Lanczos iterations and each state $(J \neq 0)$ in the parent nucleus is connected to 99 states in the daughter nucleus. Such treatment is more precise and complicated. But we found the electron capture rate by adopting LMP's GT resonance transition location and strength is close to their detailed results, so here we can estimate the effect of screening by the Brink hypothesis.

\section{Conclusions}

From the results of ${ }^{56} \mathrm{Co}\left(\mathrm{e}^{-}, v_{\mathrm{e}}\right)^{56} \mathrm{Fe}$, we can from Table 1 find that the screening factors decrease with density when $\rho<$ $10^{9} \mathrm{~g} / \mathrm{cm}^{3}$. At $\rho \approx 10^{9} \mathrm{~g} / \mathrm{cm}^{3}$, the screening factor reaches its minimum for ${ }^{56} \mathrm{Co}$, which indicates that the screening effect is at the maximum in this case. Comparing to Aufderheide's results, the screening effect on the improved electron capture rates is decreased at low density $\left(C_{2}>C_{1}\right)$ and enhanced at higher density $\left(10^{9} \mathrm{~g} / \mathrm{cm}^{3} \leq \rho Y_{\mathrm{e}} \leq 10^{11} \mathrm{~g} / \mathrm{cm}^{3}\right)$. The GT resonance transition mainly occur at high density. Larger $E_{\mathrm{GT}}$ makes the GT transition rates decrease and then it makes the screening more important so that the screening factor decrease about 1 percent. The electron capture is a decisive factor in inducing core collapse in the high-density region $\rho>10^{9} \mathrm{~g} / \mathrm{cm}^{3}$, which means the screening effect should be included at the collapse stage of presupernova. Table 2 shows the screening factors in densities $\rho Y_{\mathrm{e}}=10^{9} \mathrm{~g} / \mathrm{cm}^{3}$ and $\rho Y_{\mathrm{e}}=10^{10} \mathrm{~g} / \mathrm{cm}^{3}$. It shows that the higher the temperature, the closer to 1 the screening factor is (i.e. the effect of screening is closer to zero). The reason is that when the temperature is higher, the average electron energy is higher than the screening potential. It can also be found that the influence of temperature is low because the Fermi energy of the electron is decided by density. Screening factors stay about 0.9 when $\rho Y_{\mathrm{e}}>10^{9} \mathrm{~g} / \mathrm{cm}^{3}$ for ${ }^{56} \mathrm{Co}$ (i.e. the influence of screening is about 10 percent). We expect the other nuclei have similar results to ${ }^{56} \mathrm{Co}$ since their corrections to the GT transition are similar (LMP 2000, 1999). Presupernova structure and the subsequent evolution of collapse process may be modified due to the screening effect on electron capture. Theoretically, screening will decrease the collapse velocity, prolong the collapse timescale, increase the loss of neutrino energy, and so on. But there are many feedback effects in the numerical simulations, so these effects need to be confirmed by detailed numerical simulations.

Acknowledgements. This work was supported by the National Natural Science Foundation of China under Grant No. 10347008. One of the authors (Menquan Liu) would like to thank Bin Wang at Peking University for useful discussions and help with preparing this paper. 


\section{References}

Aufderheide, M. B., Fushiki, I., Woosley, S. E., et al. 1994, ApJS, 91, 389 Bethe, H. A. 1990, Rev. Mod. Phys., 62, 801

Bethe, H. A., Brown, G. E., Applegate, J., et al. 1979, Nucl. Phys. A, 324, 487 Bravo, E., \& Garcia-Senz, D. 1999, MNRAS, 307, 984

Chen, F. S., Durand, L., \& McGee, I. J. 1966, Phys. Rev., 146, 638 Fuller, G. M., Fowler, W. A., \& Newman, M. J. 1982, ApJ, 252, 715

Fuller, G. M., Fowler, W. A., \& Newman, M. J. 1982, ApJS, 48, 279

Gutierrez, J., Garcia-Berro, E., Iben, I., \& Isern, J. 1996, ApJ, 459, 701

Heger, A., Woosley, S. E., Martinez-Pinedo, G., et al. 2001, ApJ, 560, 325
Itoh, N., Tomizawa, N., Tamamura, M., et al. 2002, ApJ, 579, 380 Kar, K., Ray, A., \& Sarkar, S. 1994, ApJ, 434, 662

Koonin, S. E., Dean, D. J., \& Langanke, K. 1997, Phys. Rep., 278, 1 Langanke, K., \& Martinez-Pinedo, G. 1999, Phys. Lett. B, 453, 187 Langanke, K., \& Martinez-Pinedo, G. 2000, Nucl. Phys. A, 673, 481 Luo, Z. Q., \& Peng, Q. H. 1996, Science in China (Series A), 39, 776 Luo, Z. Q., \& Peng, Q. H. 2001, ChAA, 25, 1

Matese, J. J., \& John, W. R. 1966, Phys. Rev., 150, 846

Pruet, J., \& Fuller, G. M. 2003, ApJS, 149, 189

Reitz, J. R. 1950, Phys. Rev., 77, 10

Salpeter, E. E., \& Van Horn, H. M. 1969, ApJ, 155, 183

Woosley, S. E., Heger, A., \& Weaver, T. A. 2002, Rev. Mod. Phys., 74, 1015 